\title{
Optical study of local strain related disordering in CVD-grown $\mathrm{MoSe}_{2}$ monolayers
}

\author{
J. Krustok, ${ }^{1, \text { a) }}$ T. Raadik, ${ }^{1}$ R. Jaaniso, ${ }^{2}$ V. Kiisk, ${ }^{2}$ I. Sildos, ${ }^{2}$ M. Marandi, ${ }^{2}$ H.-P. Komsa, ${ }^{3}$ \\ B. Li, ${ }^{4}$ X. Zhang, ${ }^{5}$ Y. Gong, ${ }^{5, b)}$ and P. M. Ajayan ${ }^{5}$ \\ ${ }^{1}$ Department of Materials Science, Tallinn University of Technology, Ehitajate tee 5, 19086 Tallinn, Estonia \\ ${ }^{2}$ Institute of Physics, University of Tartu, W. Ostwaldi 1, 50411 Tartu, Estonia \\ ${ }^{3}$ COMP, Department of Applied Physics, Aalto University, P.O. Box 11100, 00076 Aalto, Finland \\ ${ }^{4}$ Department of Mechanical Engineering, Villanova University, 800 Lancaster Avenue, Villanova, \\ Pennsylvania 19085, USA \\ ${ }^{5}$ Department of Chemistry, Rice University, Houston, Texas 77005, USA
}

(Received 27 September 2016; accepted 8 December 2016; published online 20 December 2016)

\begin{abstract}
We present temperature dependent micro-photoluminescence and room temperature photoreflectance spectroscopy studies on aged $\mathrm{MoSe}_{2}$ monolayers with high surface roughness. $\mathrm{A}^{0}$ and $\mathrm{B}^{0}$ exciton bands were detected at $1.512 \mathrm{eV}$ and $1.72 \mathrm{eV}$, respectively, which are $50-70 \mathrm{meV}$ lower than those commonly reported for high-quality samples. It is shown that the difference can be accounted for using a model of localized excitons for disordered $\mathrm{MoSe}_{2}$ monolayers where the optical band gap energy fluctuations could be caused by random distribution of local tensile strain due to surface roughness. The density of localized exciton states is found to follow the Lorentzian shape, where the peak of this distribution is about $70 \mathrm{meV}$ from the energy of delocalized states. Published by AIP Publishing. [http://dx.doi.org/10.1063/1.4972782]
\end{abstract}

Two-dimensional (2D) semiconductor films, such as transition metal dichalcogenides (TMD), $\mathrm{MoS}_{2}, \mathrm{MoSe}_{2}, \mathrm{WS}_{2}$, and $\mathrm{WSe}_{2}$, have become as a new group of materials with remarkable electrical and optical properties. ${ }^{1-5}$ Generally, these bulk materials are indirect semiconductors whereas a direct optical band gap emerges when their thickness is reduced from a few layers to a single layer. The direct band gap in single layer TMDs usually results in an intense excitonic photoluminescence (PL) and also provides different prospects in optoelectronic applications.

Recently, monolayer $\mathrm{MoSe}_{2}$ has started to gain more and more attention because it has a direct optical band gap about $1.58 \mathrm{eV}$ at room temperature and is therefore an attractive material for solar cells and near-IR photodetectors. ${ }^{6}$ Monolayer $\mathrm{MoSe}_{2}$ is actually a "three-layer" structure where the Mo layer is sandwiched between top and bottom Se layers. Moreover, monolayer $\mathrm{MoSe}_{2}$ has a large spin-splitting energy of $\sim 180 \mathrm{meV}$ at the top of the valence bands, which makes $\mathrm{MoSe}_{2}$ applicable also in spintronics. ${ }^{7}$ Optical properties of $\mathrm{MoSe}_{2}$ with different numbers of monolayers were studied in many papers. ${ }^{8-16}$ The room temperature photoluminescence (PL) spectrum of the $\mathrm{MoSe}_{2}$ monolayer is characterized by the presence of two exciton peaks that arise from vertical transitions at the $\mathrm{K}$ point of the Brillouin zone from a spin-orbit-split valence band to a nearly degenerate conduction band. ${ }^{6}$ These PL bands are called as A and B bands, and the peak positions of these bands are near $1.56-1.58$ and $1.76-1.78 \mathrm{eV}$, respectively. ${ }^{10,11}$ However, the peak positions of PL bands and also their shape depend on the layer preparation method. Often, monolayers grown by chemical vapor deposition (CVD) on $\mathrm{Si} / \mathrm{SiO}_{2}$ substrates show PL emission at

\footnotetext{
${ }^{a)}$ E-mail: Juri.Krustok@ttu.ee

${ }^{b}$ Present address: Department of Materials Science and Engineering, Stanford University, Stanford, California 94305, USA.
}

lower energies with respect to monolayers transferred by mechanical exfoliation from bulk crystals or monolayers grown on other substrates. ${ }^{17}$ Moreover, it was proposed in many papers that at room temperature the A exciton peak is actually a sum of a neutral $\mathrm{A}^{0}$ exciton and a weaker charged exciton (trion) $\mathrm{A}^{\prime}$ at lower photon energy causing an asymmetrical shape of the A band. At low temperatures, these exciton peaks are clearly resolved and the trion peak dominates. ${ }^{10}$ The separation between $\mathrm{A}^{0}$ and $\mathrm{A}^{\prime}$ is about $30 \mathrm{meV}$ in $\mathrm{MoSe}_{2}$ monolayers. ${ }^{10}$ The $\mathrm{B}$ band is usually related to a neutral $\mathrm{B}^{0}$ exciton and it is located at about $180-200 \mathrm{meV}$ higher energy than the $\mathrm{A}^{0}$ exciton due to spin-orbit splitting of the valence band. The $\mathrm{B}^{0}$ peak is very weak (even at low temperatures) and therefore it is always a challenge to get reliable information about $\mathrm{B}$ excitons.

The red-shift of PL bands in CVD grown $\mathrm{MoSe}_{2}$ could be explained by a tensile strain in a monolayer. Recent experiments showed that one percent of uniaxial strain increase gives a reduction of $\sim 42 \mathrm{meV}$ in the A-exciton gap in monolayer $\mathrm{MoSe}_{2} \cdot{ }^{18}$ The strain can be created due to different thermal expansion coefficients (TECs) of the $\mathrm{SiO}_{2}$ substrate and $\mathrm{MoSe}_{2}$. Another explanation of this red-shift is related to the surface roughness which is causing local strain depending on a surface topography. In $\mathrm{MoS}_{2}$ monolayers, this concept was also verified by creating artificial atoms ${ }^{19}$ or wrinkles ${ }^{20}$ on a substrate surface, thus getting very high values of local tensile strain. All these elevated $\mathrm{MoS}_{2}$ regions showed tensile strain up to $2.5 \%$ and optical band gap values about $70-90 \mathrm{meV}$ less than the unaffected monolayer, and excitons tend to funnel and localize in these areas showing approximately 2 times higher PL intensity. On the other hand, it was shown that the surface roughness of the $\mathrm{SiO}_{2}$ substrate may lead to considerable bending of the $\mathrm{MoS}_{2}$ sheet on top, with local strain values reaching $\pm 6 \%$ and consequently large changes in the band gap, ${ }^{21}$ although only a 
little change was observed in the PL peak position. We note that if the surface roughness can induce such strong bending, to overcome the related energy cost, then the sheet must be quite strongly bound to the substrate. In the case of high values of surface roughness, where randomly distributed elevated areas create random strain and band gap fluctuations and, as a result, we will have a typical disordered semiconductor, where the overall shape of a localized excitons PL band is largely affected by spatial band gap fluctuations. A high value of surface roughness can be caused by aging processes, when usually large-scale structural and morphological changes can be detected due to the gradual oxidation of grain boundaries or defects. ${ }^{22}$ However, disordering in aged monolayers could be related also to local distribution of charged defects or structural imperfections. Unfortunately, optical properties of aged TMD monolayers are still not well known.

In this work, we study the optical properties of CVDgrown and aged $\mathrm{MoSe}_{2}$ monolayers having quite high surface roughness.

$\mathrm{MoSe}_{2}$ monolayers were grown via CVD from $\mathrm{MoO}_{3}$ and selenium on a $\mathrm{Si}$ substrate with a $275 \mathrm{~nm}$ thick $\mathrm{SiO}_{2}$ layer at $750{ }^{\circ} \mathrm{C}$. This growth process produced uniform $\mathrm{MoSe}_{2}$ domains, and the size of these areas ranges from several tens to more than hundred micrometers. More detailed description of $\mathrm{MoSe}_{2}$ growth can be found in the paper by Wang et al. ${ }^{23}$ All measurements were performed on aged (about 6 month) samples.

Raman, reflectance contrast, and $\mu \mathrm{PL}$ measurements were carried out using a Horiba LabRAM HR800 MicroRaman system or a Renishaw inVia Raman microscope (through $50 \times$ and $100 \times$ objective lenses) equipped with a multichannel CCD detection system in the backscattering configuration with a spectral resolution better than $1 \mathrm{~cm}^{-1}$. A Nd-YAG laser (wavelength $532 \mathrm{~nm}$ ) or an Ar laser (wavelength $488 \mathrm{~nm}$ ) was used for excitation. Linkam THMS350V heating/cooling stage was used for low temperature PL measurements. PL imaging was carried out on a separate microscope unit equipped with a high-power $505 \mathrm{~nm}$ light emitting diode (Thorlabs M505L3) for wide-field excitation and a cooled Atik 414EX CCD camera for detection (proper dichroic beamsplitter as well as excitation and emission filters were employed).

Atomic force microscopy (AFM) experiments were carried out with a multimode AFM Autoprobe CP II (Veeco). All images were recorded in the non-contact mode using HAHR (NT-MDT) series cantilevers under ambient conditions. The Gwyddion free software ver. 2.42 (Czech Metrology Institute) was employed for image processing using the firstorder flattening for background slope removal. If necessary, the contrast and brightness were adjusted.

Photoreflectance (PR) measurements were performed at room temperature using a $40 \mathrm{~cm}$ grating monochromator and a $250 \mathrm{~W}$ halogen lamp as the primary beam and a modulated $50 \mathrm{~mW}$ He-Cd laser (Kimmon, $\lambda=442 \mathrm{~nm}$ ) as the secondary beam. The spot size was about $500 \mu \mathrm{m}$. The PR signal at $85 \mathrm{~Hz}$ was detected using a Si detector and a DSP lock-in amplifier. $^{24}$

CVD-grown $\mathrm{MoSe}_{2}$ monolayers show relatively large areas with a diameter over $50 \mu \mathrm{m}$ (Figure 1(a)). All these areas have quite similar Raman and PL spectra indicating that their thickness and macroscopic properties must be quite uniform. The actual thickness of these layers was determined by AFM and was less than $1 \mathrm{~nm}$, typical for $\mathrm{MoSe}_{2}$ monolayers. ${ }^{16,19,23}$ However, the root mean square (RMS) roughness of the monolayer, determined from the AFM scan, was in the range of $0.40-0.43 \mathrm{~nm}$. This value is definitely very high and we can expect some disordering related to local strain. Moreover, PL imaging revealed that the PL intensity changed more than 3 times at different locations on the monolayer (Figure 1(b)). Some small areas and sometimes also the edges of a monolayer showed an enhanced PL intensity.

The Raman spectrum of the $\mathrm{MoSe}_{2}$ monolayer is presented in Figure 2(a). Three typical Raman active modes, i.e.,

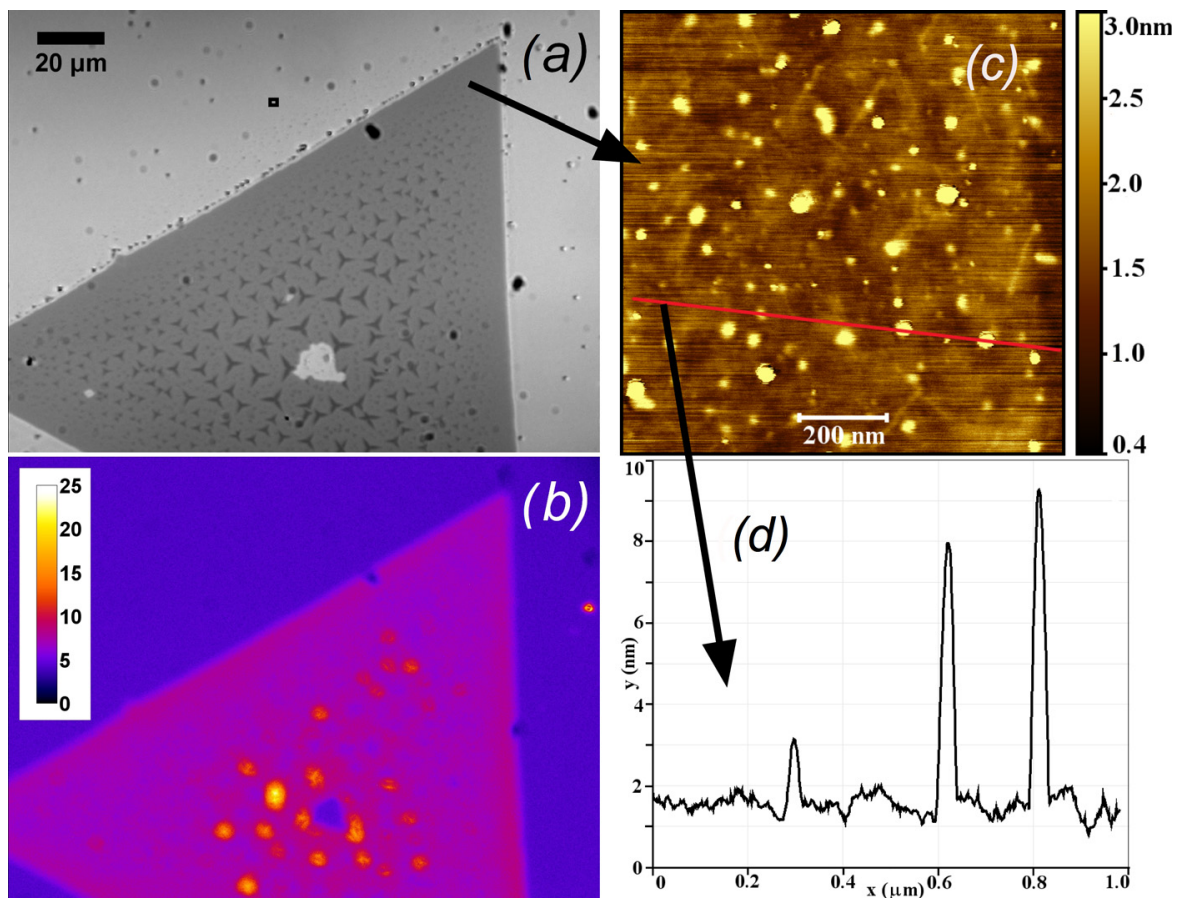

FIG. 1. Optical (a) and false color photoluminescence (b) images of a $\mathrm{MoSe}_{2}$ monolayer. The AFM image taken from the marked monolayer region (c) and a corresponding linear scan (d). 

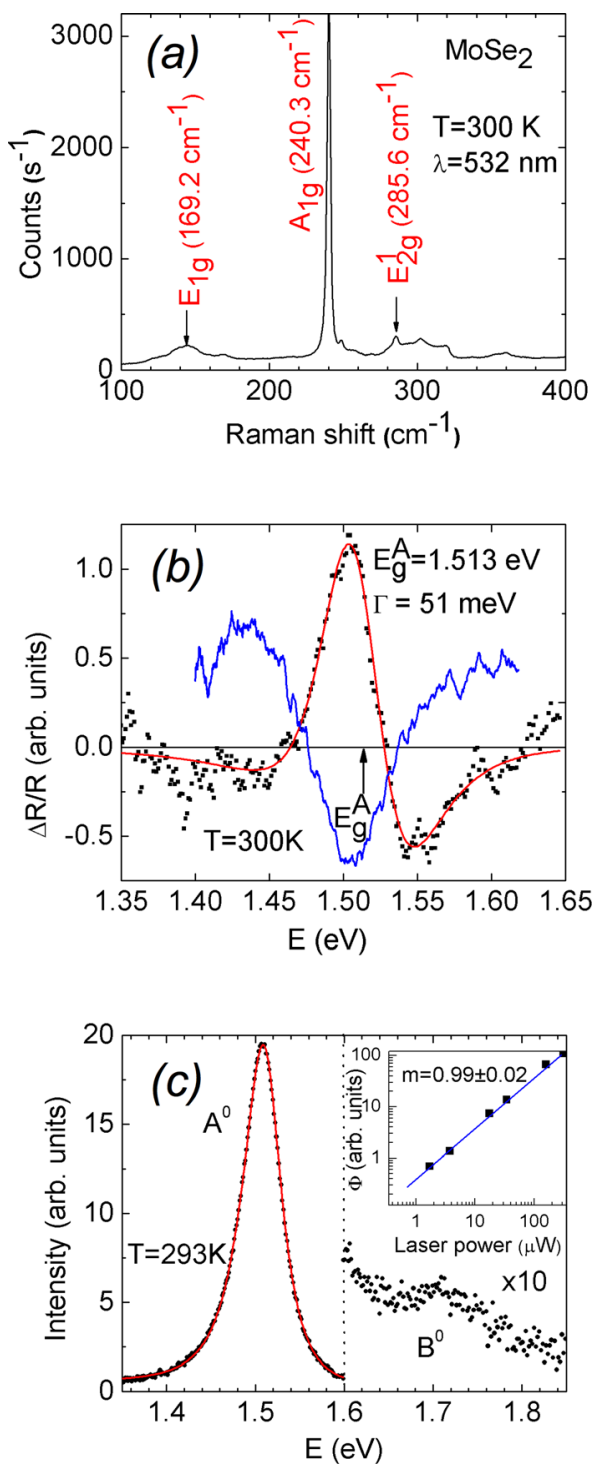

FIG. 2. Room temperature optical properties of the $\mathrm{MoSe}_{2}$ monolayer. (a) Raman spectrum, (b) PR (dots) and fitting result (red line) and reflectance contrast (blue line) spectra, and (c) PL spectrum (dots) showing also a fitting result (red line) with Eq. (1); the inset shows the $\mathrm{A}^{0}$-band integral intensity dependence on laser power.

the prominent $A_{1 g}$ Raman mode and the weaker $E_{1 g}$ and $E_{2 g}$ Raman modes, are observed in the Raman spectra. In general, the location of Raman modes can be used to determine the thickness of layers. In this study, the $A_{1 g}$ and $E_{2 g}$ modes of single-layer $\mathrm{MoSe}_{2}$ are located at $240.3 \mathrm{~cm}^{-1}$ (full width at half maximum FWHM $=2.5 \mathrm{~cm}^{-1}$ ) and $285.6 \mathrm{~cm}^{-1}$, respectively (Figure 2(a)). The spectral spacing between the $E_{2 g}$ and $\mathrm{A}_{1 \mathrm{~g}}$ modes is another essential marker of the thickness of layers, although different substrates and strains may have a certain effect. In our case, the peak spacing between $E_{2 g}$ and $\mathrm{A}_{1 \mathrm{~g}}$ is $45.3 \mathrm{~cm}^{-1}$. According to Xia et al., ${ }^{8}$ the spacings are $46.9 \mathrm{~cm}^{-1}$ and $42 \mathrm{~cm}^{-1}$ for $\mathrm{MoSe}_{2}$ monolayers and thicker layers, respectively. This result also confirms the presence on $\mathrm{MoSe}_{2}$ monolayers although the spacing in our sample is slightly smaller. This could be a result of randomly distributed tensile strain in our CVD-grown films. It was shown that in CVD-grown strained $\mathrm{WS}_{2}$ monolayers the spacing between $\mathrm{E}_{2 \mathrm{~g}}$ and $\mathrm{A}_{1 \mathrm{~g}}$ Raman modes will also be smaller than in strain free monolayers. ${ }^{17}$
Room temperature photoreflectance (PR) of our $\mathrm{MoSe}_{2}$ monolayers was also measured. Unfortunately, the experimental setup did not allow us to use very small spot size, and therefore the signal was extremely weak. We did not detect any features in the B-exciton region at room temperature, and only the A-exciton region was studied. PR spectra near the fundamental excitonic band gap energy with a weak field approach can be well fitted with a third derivative functional form given by Aspnes: ${ }^{25} \Delta R / R=\operatorname{Re}\left[C e^{i \varphi}\left(E-E_{g}+i \Gamma\right)^{-n}\right]$, where $E$ is the photon energy, $C$ is an amplitude parameter, $\varphi$ is the phase parameter, $E_{g}$ is the energy of the optical bandgap, and $\Gamma$ is a broadening parameter. The exponent $n$ depends on the type of the critical point and is usually related to the line shape of the dielectric function (e.g., Lorentzian or Gaussian). The best fitting was achieved using the 2D critical point approach, i.e., $n=3$, see Figure 2(b). The obtained room temperature optical band gap energy $E_{g}{ }^{A}=1.513 \mathrm{eV}$ is relatively small, because usually values around $1.55-1.58 \mathrm{eV}$ were reported. ${ }^{8-10,16,26}$ The broadening parameter $\Gamma=51 \mathrm{meV}$ is also higher than that measured in Ref. 9, but the difference is smaller than for band gap energy. A similar result was found also from the reflectivity contrast measurement, where spectral differences of reflectivity between the $\mathrm{Si} / \mathrm{SiO}_{2}$ substrate and $\mathrm{MoSe}_{2}$ monolayer were measured using the method described in Ref. 9, see Figure 2(b) (blue line). We could not detect any features near the band gap energy of unstrained $\mathrm{MoSe}_{2}(\sim 1.58 \mathrm{eV}$ (Ref. 9)), and this fact confirms that our PR band is not related to defect-bound excitons.

Room temperature $\mu \mathrm{PL}$ spectra are shown in Figure 2(c). $\mathrm{A}^{0}$ and $\mathrm{B}^{0}$ bands were detected at $1.512 \mathrm{eV}$ and $1.72 \mathrm{eV}$, respectively. The $\mathrm{A}^{0}$ band showed significant asymmetric broadening on the lower energy (LE) side and its integral intensity $\Phi$ has almost linear dependence on laser power $P: \Phi \sim P^{0.99}$, see the inset of Figure 2(c). This intensity behavior is typical for excitonic PL and we did not detect any significant changes of the shape of $\mathrm{A}^{0} \mathrm{PL}$ band with increasing laser power. Therefore, we ruled out a possibility that the asymmetry of the $\mathrm{A}^{0}$ band is caused by a trion or biexciton emission ${ }^{27}$ or by defect-bound excitons.

The temperature dependence of the $\mathrm{A}^{0}$ band is presented in Figure 3. At lower temperatures, the $\mathrm{A}^{0}$ band shows a blue shift and its asymmetry is more prominent. We also observed a weak PL band (X-band) at about $1.46 \mathrm{eV}$ at temperatures $\mathrm{T}<100 \mathrm{~K}$, probably related to deeper defect states. The PL peak integral intensity decreases with temperature according to the exponential law $I(T)=I_{0} /\left[1+\alpha \exp \left(E_{T} / k T\right)\right]$ with $E_{T}=48 \mathrm{meV}$, see Figure 3(d). The thermal quenching of the PL band is the result of the interplay between excitonic radiative recombination from the localized excitons and nonradiative recombination. The temperature dependence of peak positions for both exciton bands follows the trend of $E_{g}{ }^{0}$ measured in Ref. 9 for strain-free exfoliated from bulk crystals $\mathrm{MoSe}_{2}$ monolayers, but the $\mathrm{A}^{0}$ band is red-shifted about $70 \mathrm{meV}$, see Figure 3(b). At the same time, the separation between $\mathrm{A}^{0}$ and $\mathrm{B}^{0}$ bands is almost constantly $200 \mathrm{meV}$, similar to recent low temperature data. ${ }^{28}$ The splitting arises from the combined effect of spin-orbit splitting of the valence band by $180 \mathrm{meV}$ and of the conduction band by $21 \mathrm{meV} .{ }^{28} \mathrm{We}$ also observe that the FWHM of a low energy side does not change with temperature (see Figure 3(c)) 

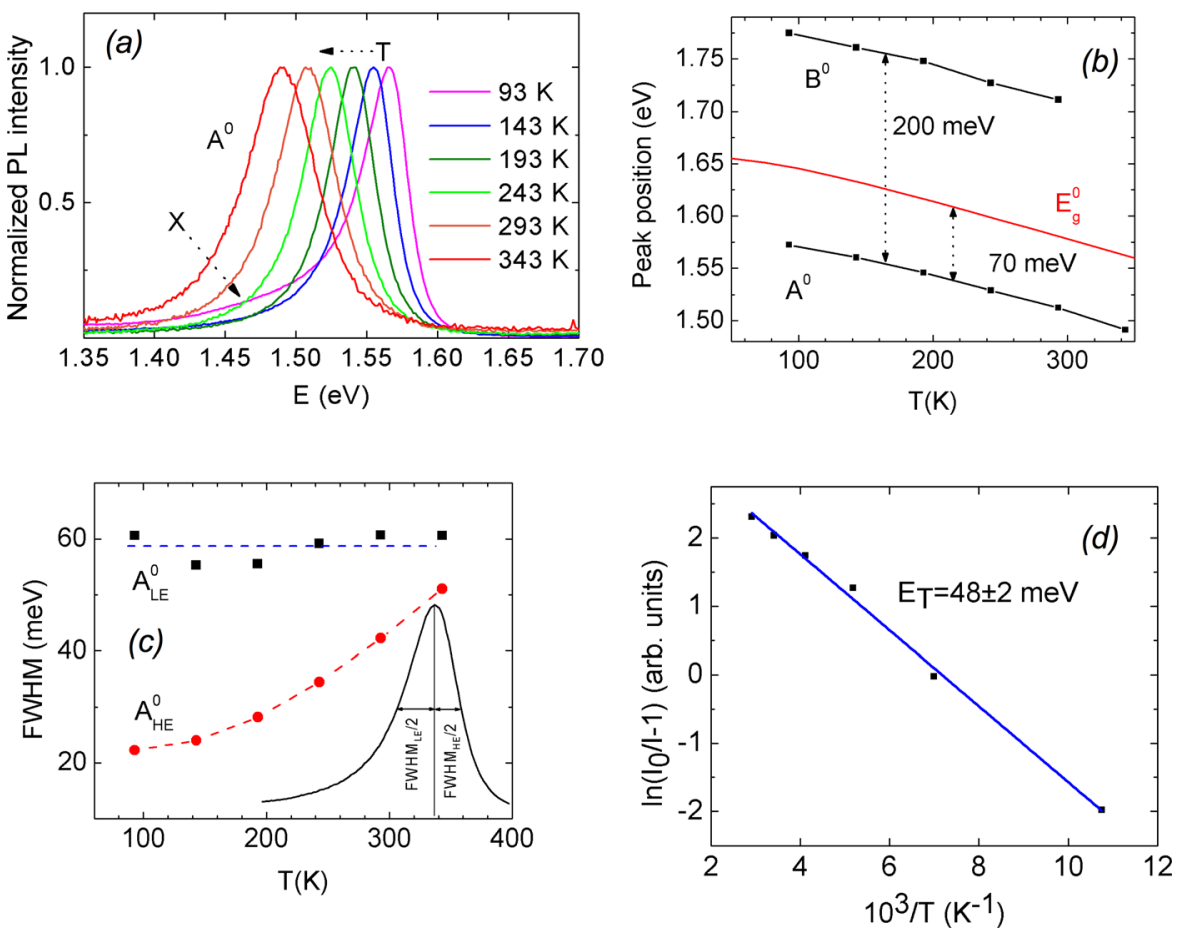

FIG. 3. Temperature dependence of PL bands in the $\mathrm{MoSe}_{2}$ monolayer: (a) normalized PL spectra, (b) peak positions of $\mathrm{A}^{0}$ and $\mathrm{B}^{0}$ exciton peaks, red line represents a temperature dependence of $\mathrm{E}_{\mathrm{g}}{ }^{0}$ taken from Ref. 9, (c) FWHM values for low-energy (LE) and high-energy (HE) side of the $\mathrm{A}^{0}$ exciton band, and (d) integral intensity of the $\mathrm{A}^{0}$ band (dots) and a fitting (line) showing an activation energy $E_{T}=48 \mathrm{meV}$. while the FWHM of a high energy (HE) side shows a certain temperature dependence. The FWHM of the $\mathrm{A}^{0}$ exciton band is then a sum $\mathrm{FWHM}=\mathrm{FWHM}_{\mathrm{LE}} / 2+\mathrm{FWHM}_{\mathrm{HE}} / 2$. At higher temperatures, the $\mathrm{FWHM}_{\mathrm{HE}}$ is very close to the width of the symmetrical $\mathrm{A}^{0}$ exciton band measured in Ref. 9, but at low temperatures the role of an additional broadening increases. This kind of behavior is typical for disordered samples where the shape of the low-energy side of the PL band is determined by the density of localized states function $\rho(E)$ and the shape of high-energy side is mainly due to the temperature dependent distribution function $f(E, T)$ of localized carriers. These localized states can be formed by potential fluctuations caused by a high concentration of charged defects, ${ }^{29,30}$ by spatial variation of semiconductor alloy composition, ${ }^{31,32}$ or by variation of quantum well and quantum dot properties. ${ }^{33,34}$ In many cases, the $\rho(E)$ function has a Gaussian shape, but other shapes are also encountered. The distribution function $f(E, T)$ has more complicated shape and for localized states with possible thermal escape and recapture of carriers this function was studied by Li et al. ${ }^{34}$ According to this work, the $f(E, T)$ function has a shape resembling a Fermi distribution with a characteristic energy $E_{a}$ representing the energetic position of a delocalized state to which the localized carriers can thermally escape. It was also shown that generally at lower temperatures the distribution function does not modify the lowenergy side of the PL band and therefore by examining the shape of this side it will be possible to find $\rho(E)$. In our case, the Lorentzian shape was dominating the low-energy side of the $\mathrm{A}^{0}$ band. Accordingly, the final shape of our $\mathrm{A}^{0} \mathrm{PL}$ band is found to be

$$
\begin{aligned}
I(E, T) & =A(T) \rho(E) f(E, T) \\
& =\frac{A(T) \rho_{0} \tau_{t r} / \tau_{r}}{\left[1+\left(\frac{E-E_{0}}{W}\right)^{2}\right] \exp \left[\frac{E-E_{a}}{k T}\right]+\tau_{t r} / \tau_{r}},
\end{aligned}
$$

where $A(T)$ is a temperature dependent term, $\rho_{0}$ is the amplitude, $E_{0}$ is a peak position, $W$ is a width of the density of states function, and $1 / \tau_{r}$ and $1 / \tau_{t r}$ represent the rate of radiative recombination and the attempt-to-escape rate of the localized carriers, respectively. ${ }^{34} \mathrm{We}$ assume that delocalized states are responsible for the $\mathrm{A}^{0}$ exciton emission in unstrained $\mathrm{MoSe}_{2}$ layers and thus, according to Figure 3(b), $\mathrm{E}_{\mathrm{a}}=\mathrm{E}_{0}+70 \mathrm{meV}$. Fitting the shape of our PL band with Eq. (1) gave very good results at all temperatures. One example of this fitting is given in Figure 2(c), where values $W=23 \mathrm{meV}, E_{0}=1.510 \mathrm{eV}$, and $\tau_{t r} / \tau_{r}=0.4$ were found. The FWHM of the density of states function $2 W=46 \mathrm{meV}$ is very close to the value of the broadening parameter $\Gamma$ found from PR measurements. The obtained form for $\rho(E)$ entails that there is considerable average strain in addition to the random strain fluctuations. We propose that this is due to the different thermal expansion coefficients (TECs). Adopting TEC values of $0.55 \times 10^{-6} / \mathrm{K}^{35} 7.24 \times 10^{-6} / \mathrm{K}$, and $1.9 \times 10^{-6} / \mathrm{K}$ (Ref. 36) for $\mathrm{SiO}_{2}, \mathrm{MoSe}_{2}$, and $\mathrm{MoS}_{2}$, and $750^{\circ} \mathrm{C}$ difference between growth and room temperatures, the resulting tensile strains are $0.5 \%$ for $\mathrm{MoSe}_{2}$ and $0.1 \%$ for $\mathrm{MoS}_{2}$ on the $\mathrm{SiO}_{2}$ substrate. According to first-principles calculations, biaxial strain leads to a band gap decrease of $90 \mathrm{meV}$ per percent of strain, ${ }^{26}$ and thus $45 \mathrm{meV}$ for room temperature in our case. At lower temperatures, the strain can be even larger, and thus lends support that the $70 \mathrm{meV}$ shift arises largely from the residual strain due to the TEC difference. The $\mathrm{MoS}_{2}$ value $0.1 \%$ is in good agreement with the additional strain found in Ref. 21 in the case of $\mathrm{MoS}_{2}$ on $\mathrm{SiO}_{2}$. Since the subsequent optical band gap change is only in the order of $10 \mathrm{meV}$, such a dramatic shift in the A-peak position in $\mathrm{MoS}_{2}$ is not expected.

In conclusion, all the obtained results confirm that the model of localized excitons is valid for disordered $\mathrm{MoSe}_{2}$ monolayers where the optical band gap energy fluctuations could be caused by random distribution of local tensile strain 
due to surface roughness. The density of localized excitons is found to follow the Lorentzian shape, where the peak of this distribution is about $70 \mathrm{meV}$ from the energy of delocalized states. This energy shift is proposed to originate mainly from the strain due to different thermal expansion coefficients of the substrate and the $\mathrm{MoSe}_{2}$ layer.

This work was supported by institutional research funding IUT 19-28 and IUT 34-27 of the Estonian Ministry of Education and Research, by the European Union through the European Regional Development Fund (Project TK141), and by FP7 project CHEETAH, EC Grant Agreement No. 609788. H.-P.K. acknowledges support from the U.S. Army RDECOM via Contract No. W911NF-15-1-0606, Academy of Finland through Project Nos. 263416 and 286279, and Centres of Excellence Programme (2012-2017) under Project No. 251748.

${ }^{1}$ G. R. Bhimanapati, Z. Lin, V. Meunier, Y. Jung, J. Cha, S. Das, D. Xiao, Y. Son, M. S. Strano, V. R. Cooper, L. Liang, S. G. Louie, E. Ringe, W. Zhou, S. S. Kim, R. R. Naik, B. G. Sumpter, H. Terrones, F. Xia, Y. Wang, J. Zhu, D. Akinwande, N. Alem, J. A. Schuller, R. E. Schaak, M. Terrones, and J. A. Robinson, ACS Nano 9, 11509 (2015).

${ }^{2}$ Y. Gong, S. Lei, G. Ye, B. Li, Y. He, K. Keyshar, X. Zhang, Q. Wang, J. Lou, Z. Liu, R. Vajtai, W. Zhou, and P. M. Ajayan, Nano Lett. 15, 6135 (2015).

${ }^{3}$ M. M. Ugeda, A. J. Bradley, S.-F. Shi, F. H. da Jornada, Y. Zhang, D. Y. Qiu, S.-K. Mo, Z. Hussain, Z.-X. Shen, F. Wang, S. G. Louie, and M. F. Crommie, Nat. Mater. 13, 1091 (2014).

${ }^{4}$ C. N. R. Rao and U. Maitra, Annu. Rev. Mater. Res. 45, 29 (2015).

${ }^{5}$ C. Tan and H. Zhang, Chem. Soc. Rev. 44, 2713 (2015).

${ }^{6}$ Y. Zhang, T.-R. Chang, B. Zhou, Y.-T. Cui, H. Yan, Z. Liu, F. Schmitt, J. Lee, R. Moore, Y. Chen, H. Lin, H.-T. Jeng, S.-K. Mo, Z. Hussain, A. Bansil, and Z.-X. Shen, Nat. Nanotechnol. 9, 111 (2014).

${ }^{7}$ A. Ramasubramaniam, Phys. Rev. B 86, 115409 (2012).

${ }^{8}$ J. Xia, X. Huang, L.-Z. Liu, M. Wang, L. Wang, B. Huang, D.-D. Zhu, J.-J. Li, C.-Z. Gu, and X.-M. Meng, Nanoscale 6, 8949 (2014).

${ }^{9}$ A. Arora, K. Nogajewski, M. Molas, M. Koperski, and M. Potemski, Nanoscale 7, 20769 (2015).

${ }^{10}$ J. S. Ross, S. Wu, H. Yu, N. J. Ghimire, A. M. Jones, G. Aivazian, J. Yan, D. G. Mandrus, D. Xiao, W. Yao, and X. Xu, Nat. Commun. 4, 1474 (2013).

${ }^{11}$ X. Lu, M. I. B. Utama, J. Lin, X. Gong, J. Zhang, Y. Zhao, S. T. Pantelides, J. Wang, Z. Dong, Z. Liu, W. Zhou, and Q. Xiong, Nano Lett. 14, 2419 (2014).
${ }^{12}$ A. Branny, G. Wang, S. Kumar, C. Robert, B. Lassagne, X. Marie, B. D. Gerardot, and B. Urbaszek, Appl. Phys. Lett. 108, 142101 (2016).

${ }^{13}$ G. Wang, E. Palleau, T. Amand, S. Tongay, X. Marie, and B. Urbaszek, Appl. Phys. Lett. 106, 112101 (2015).

${ }^{14}$ P. Tonndorf, R. Schmidt, P. Böttger, X. Zhang, J. Börner, A. Liebig, M. Albrecht, C. Kloc, O. Gordan, and D. R. Zahn, Opt. Express 21, 4908 (2013).

${ }^{15}$ H.-V. Han, A.-Y. Lu, L.-S. Lu, J.-K. Huang, H. Li, C.-L. Hsu, Y.-C. Lin, M.-H. Chiu, K. Suenaga, C.-W. Chu, H.-C. Kuo, W.-H. Chang, L.-J. Li, and Y. Shi, ACS Nano 10, 1454 (2016).

${ }^{16}$ J. C. Shaw, H. Zhou, Y. Chen, N. O. Weiss, Y. Liu, Y. Huangand, and X. Duan, Nano Res. 7, 511 (2014).

${ }^{17}$ L. Su, Y. Yu, L. Cao, and Y. Zhang, Nano Res. 8, 2686 (2015).

${ }^{18}$ J. Ji, A. Zhang, T. Xia, P. Gao, Y. Jie, Q. Zhang, and Q. Zhang, Chin. Phys. B 25, 077802 (2016).

${ }^{19}$ H. Li, A. W. Contryman, X. Qian, S. M. Ardakani, Y. Gong, X. Wang, J. M. Weisse, C. H. Lee, J. Zhao, P. M. Ajayan, J. Li, H. C. Manoharan, and X. Zheng, Nat. Commun. 6, 7381 (2015).

${ }^{20}$ A. Castellanos-Gomez, R. Roldan, E. Cappelluti, M. Buscema, F. Guinea, H. S. J. van der Zant, and G. A. Steele, Nano Lett. 13, 5361 (2013).

${ }^{21}$ B. G. Shin, G. H. Han, S. J. Yun, H. M. Oh, J. J. Bae, Y. J. Song, C.-Y. Park, and Y. H. Lee, Adv. Mater. 28, 9378 (2016).

${ }^{22}$ J. Gao, B. Li, J. Tan, P. Chow, T.-M. Lu, and N. Koratkar, ACS Nano 10, 2628 (2016).

${ }^{23}$ X. Wang, Y. Gong, G. Shi, W. L. Chow, K. Keyshar, G. Ye, R. Vajtai, J. Lou, Z. Liu, E. Ringe, B. K. Tay, and P. M. Ajayan, ACS Nano 8, 5125 (2014).

${ }^{24}$ T. Raadik, J. Krustok, and M. V. Yakushev, Physica B 406, 418 (2011).

${ }^{25}$ D. E. Aspnes, Surf. Sci. 37, 418 (1973).

${ }^{26}$ J. O. Island, A. Kuc, E. H. Diependaal, R. Bratschitsch, H. S. J. van der Zant, T. Heine, and A. Castellanos-Gomez, Nanoscale 8, 2589 (2016).

${ }^{27}$ I. Kylänpää and H. P. Komsa, Phys. Rev. B 92, 205418 (2015).

${ }^{28}$ G. Wang, C. Robert, A. Suslu, B. Chen, S. Yang, S. Alamdari, I. C. Gerber, T. Amand, X. Marie, S. Tongay, and B. Urbaszek, Nat. Commun. 6, 10110 (2015).

${ }^{29}$ A. P. Levanyuk and V. V. Osipov, Sov. Phys. Usp. 24, 187 (1981).

${ }^{30}$ J. Krustok, H. Collan, M. Yakushev, and K. Hjelt, Phys. Scr., T 79, 179 (1999).

${ }^{31}$ A. R. Mohmad, F. Bastiman, C. J. Hunter, R. D. Richards, S. J. Sweeney, J. S. Ng, J. P. R. David, and B. Y. Majlis, Phys. Status Solidi B 251, 1276 (2014).

${ }^{32}$ Q. Li, S. J. Xu, W. C. Cheng, M. H. Xie, S. Y. Tong, C. M. Che, and H. Yang, Appl. Phys. Lett. 79, 1810 (2001).

${ }^{33}$ J. Ma, X. Ji, G. Wang, X. Wei, H. Lu, X. Yi, R. Duan, J. Wang, Y. Zeng, J. Li, F. Yang, C. Wang, and G. Zou, Appl. Phys. Lett. 101, 131101 (2012).

${ }^{34}$ Q. Li, S. J. Xu, M. H. Xie, and S. Y. Tong, Europhys. Lett. 71, 994 (2005).

${ }^{35}$ J. W. Berthold, S. F. Jacobs, and M. A. Norton, Metrologia 13, 9 (1977).

${ }^{36}$ S. H. El-Mahalawy and B. L. Evans, J. Appl. Crystallogr. 9, 403 (1976). 